PROCEEDINGS OF THE

AMERICAN MATHEMATICAL SOCIETY

Volume 124, Number 12, December 1996, Pages 3843-3850

S 0002-9939(96)03492-2

\title{
GEODESIC NETS ON THE 2-SPHERE
}

\author{
JOEL HASS AND FRANK MORGAN \\ (Communicated by Christopher Croke)
}

\begin{abstract}
In this paper we introduce the concept of a geodesic net, an idea which plays the role among graphs that geodesics play among simple closed curves. We establish the existence of specific geodesic nets on the 2 -sphere in certain cases.
\end{abstract}

The origin of the type of problem we consider here is the famous result, first stated by Poincaré, that there is a simple geodesic on any positively curved 2-sphere. Poincaré suggested that this geodesic could be obtained by taking a shortest curve dividing the 2-sphere into two regions of equal curvature. This result was finally proved by Croke $[\mathrm{Cr}]$, and a simple proof was given in $[\mathrm{H}-\mathrm{M}]$. Here we consider more general results of this kind.

A sample problem is the following. Call a graph with three arcs all meeting at two vertices a $\theta$ graph. On the standard round 2 -sphere, such a graph can be realized with three great semicircles of longitude, each running from the south to the north pole, and meeting at the poles at $2 \pi / 3$ angles (see Figure 1 ). This graph has the property, shared with a geodesic, that it admits no length decreasing local perturbations.

We investigate here questions such as whether such a configuration exists in other metrics on the 2-sphere.

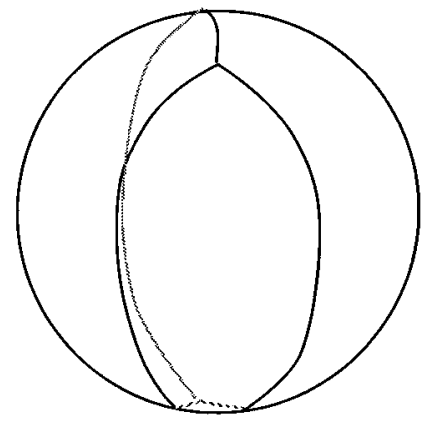

FiguRE 1. A geodesic net of three arcs meeting at 120 degrees

A graph that admits no length decreasing local perturbations consists of geodesic arcs whose unit tangent vectors sum to zero at each vertex. For a degree-three

Received by the editors January 26, 1995 and, in revised form, May 30, 1995.

1991 Mathematics Subject Classification. Primary 53C22; Secondary 53A10.

Key words and phrases. Geodesics, nets.

The first author was partially supported by the National Science Foundation.

The second author was partially supported by the National Science Foundation.

3843

(C)1996 American Mathematical Society 
vertex, this implies that the arcs meet at an angle of 120 degrees. For a degree-four vertex, it implies that the opposite angles are equal, so that the graph consists of two transversely intersecting geodesic segments at the vertex.

Definition. A geodesic net is a graph embedded on a surface whose edges are geodesic arcs and whose vertices consist of arcs whose unit tangent vectors sum to zero at each vertex.

We do not require a geodesic net to be connected.

One might at first hope that an arbitrary number of regions in the sphere could be separated by a geodesic net with vertices of degree three, but for a positively-curved sphere it is easy to obtain an upper bound of 12 regions, realized by the projection of the one-skeleton of the regular dodecahedron onto the round sphere. Indeed, for any geodesic net on a positively curved sphere, with degree-three vertices, it follows from the Gauss-Bonnet formula that the net is connected and that each region is bounded by at most five edges. Now an Euler characteristic argument bounds the number of edges by 30 and the number of regions by 12 . Heppes classified all such nets on the round sphere and found ten, with $2-10$ or 12 regions ([He], used in $[\mathrm{T}]$, illustrated in $[\mathrm{A}-\mathrm{T}])$. Of course without any bound on vertex degree, the round sphere has geodesic nets with any number $k$ of regions, consisting of $k$ equally spaced great semicircles of longitude from the north to the south pole.

For a compact surface of higher genus, a geodesic net with degree-three vertices occurs as the shortest way to cut the surface open into a simply connected region (see [Ch, Introduction and Appendix], [Ho, Section 9], and [H-M, Figure 3.2(d)]). Our methods add nothing to this case.

In any smooth compact Riemannian surface, Theorem 3.2 of [M1] provides a length-minimizing way to map a given graph $G$ into the surface so that it splits the surface into regions of prescribed areas. The length-minimizing graph is embedded except that edges may bump up against each other or degenerate to points. Note that length is counted with multiplicity where edges coincide. The lengthminimizing graph consists of constant-curvature arcs meeting at vertices of $G$ with unit tangent vectors summing to zero and at a finite number of other points where the edges remain $C^{1}$. On a 2 -sphere with strictly positive curvature $R$, one can prescribe the total curvature of each region, instead of the area [M1, 3.4]. One then obtains a length-minimizing graph as before, which now consists of arcs on which the product of the curvature of the curve and the curvature of the 2 -sphere, $k R$, is constant.

Our first theorem yields a geodesic net on any positively curved 2-sphere.

Theorem 1. Let $S$ be a 2-sphere with a smooth Riemannian metric with positive curvature. There exists a geodesic net $G$ partitioning $S$ into three components having one of the types shown in Figure 2.

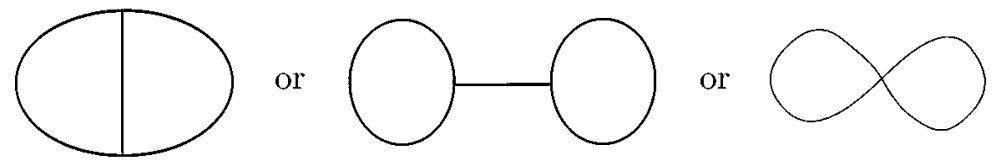

Figure 2. Three types of graphs on $S^{2}$-a $\theta$ graph, an eyeglass graph and a figure eight 
Proof. Take a graph $G$ of one of the three types shown and embed it on the 2-sphere. For each angle assign a target value $t_{i}$ by the following rules:

1. If the degree of the vertex is three, $t_{i}=2 \pi / 3$.

2. If the degree of the vertex is four, $\pi / 3 \leq t_{i} \leq 2 \pi / 3$ and $t_{i}=t_{j}$ for opposite angles.

Having assigned target values $t_{i}$ to each angle, assign to each region $F_{j}$ of $S^{2}-G$ a target total curvature $K_{j}$, by the formula:

$$
K_{j}=2 \pi-\sum\left(\pi-t_{i}\right)
$$

where the sum is over all target angles $t_{i}$ contained in $F_{j}$. Recall that the GaussBonnet Theorem gives that

$$
\int_{F_{j}} R+\int_{\partial F_{j}} k+\sum\left(\pi-s_{i}\right)=2 \pi,
$$

where $R$ is the Gauss curvature, $k$ is the geodesic curvature, and $s_{i}$ is the actual (as opposed to target) angle of the $i$ th vertex in $F_{j}$. If all of the edges of $G$ are geodesics and the angles $s_{i}$ at the vertices are given by the target values $t_{i}$, then the total curvature of each region is equal to its target total curvature:

$$
\int_{F_{j}} R=2 \pi-\sum\left(\pi-t_{i}\right)=K_{j} .
$$

For each region of the $\theta$ graph, the target total curvature is $4 \pi / 3$. For the eyeglass graph the target total curvatures are $5 \pi / 3$ for the monogons and $2 \pi / 3$ for the 4 gon. For the figure eight graph, the monogons have equal target total curvatures, between $4 \pi / 3$ and $5 \pi / 3$, and the 2 -gon has target total curvature between $4 \pi / 3$ and $2 \pi / 3$. In each case the target total curvatures of all regions add up to $4 \pi$.

Call the following graphs on the 2-sphere admissible:

1. An embedded $\theta$ graph with each enclosed region having total curvature $4 \pi / 3$.

2. An embedded eyeglass curve with each of the two monogon regions having total curvature $5 \pi / 3$ and the other region having total curvature $2 \pi / 3$.

3. An embedded figure eight graph with each of the monogons having equal total curvature $K, 4 \pi / 3 \leq K \leq 5 \pi / 3$, and the other region having total curvature $4 \pi-2 K$.

4. A graph obtained from one of the above types by letting edges degenerate to points and bump up against each other, as in [M1].

Theorems 3.2 and 3.4 of [M1] show that given a graph, a positively curved 2sphere and target total curvatures as above, there is a length-minimizing admissible graph which divides the two-sphere into regions with those total curvatures. Minimizing the length over all allowed target curvatures, we obtain a length-minimizing admissible graph $G_{0}$. Note that the length of a minimizing graph dividing the curvature of the sphere into a given triple of total curvatures is a continuous function of these total curvatures, so there is no problem getting a minimizer. The length-minimizing property implies that edges coming into a vertex of $G_{0}$ have unit tangent vectors which sum to zero at each vertex [M1], but not that the edges are geodesic. We will show that $G_{0}$ forms a geodesic net. 
If $G_{0}$ is embedded and a region $F_{j}$ has included angles $s_{i}$, the Gauss-Bonnet formula implies that the geodesic curvature $k$ around $\partial F_{j}$ satisfies

$$
\int_{\partial F_{j}} k=\sum\left(s_{i}-t_{i}\right)
$$

Note that only degree-four vertices make a contribution since $s_{i}=t_{i}=2 \pi / 3$ at any degree three vertex. By taking limits, we see that formula (2) holds also for admissible graphs that are not embedded.

Consider a closed path $\gamma$ crossing $G_{0}$ transversely. The first variation formula for the length of a $C^{2}$-curve implies that the derivative of the length of the curve when it is perturbed in the direction of a vector $W$ is given by the inner product of $W$ and the curvature vector of the curve. The derivative of the total curvature on one side of a curve under such a perturbation is given by the product of $R$ and the normal part of $W$. It follows that an infinitesimal push of $G_{0}$ in the direction of $\gamma$ will produce a new graph of smaller length which divides $S^{2}$ into regions of the same total curvatures unless the sum over all points where $\gamma$ crosses $G_{0}$ of $k R$ is equal to zero. Therefore we can define a real-valued pressure function $p$ on the set of regions with the property that the difference of the pressures of two regions separated by an arc $g_{0}$ of $G_{0}$ having curvature $k$ is given by $\Delta p=k R$. The function $p$ is defined to be zero on an arbitrary region, and using the formula $\Delta p=k R$ to define it successively on adjacent regions separated by an arc of multiplicity one. For two adjacent regions separated by an arc which has multiplicity $m$, we use the formula $\Delta p=m k R$. Note that if two neighboring regions have the same pressure, then the edges of the graph between them have $k=0$ and are geodesics, at least where they are not bumping up against other edges.

We now consider each of the three combinatorial types of graphs depicted in Figure 2. Suppose first that $G_{0}$ is a $\theta$ graph which has not collapsed to a figure eight. If all pressures are equal, then $G_{0}$ gives a geodesic net. Otherwise let $F_{j}$ be a region of maximal pressure. Then the geodesic curvature of $\partial F_{j}$ along any boundary arc of $F_{j}$ satisfies $k \geq 0$. By $(2), \int_{\partial F_{j}} k=0$ so that $k \equiv 0$. This implies that the pressure in the other two regions is equal to that of $F_{j}$, so that all three regions have equal pressure. Since the bounding arcs do not bump near a vertex, they start out as geodesics and never bump. Hence they remain geodesics and never bump, so that all boundary arcs are geodesics. Therefore if $G_{0}$ does not degenerate to a figure eight, then it forms a geodesic net of the type of a $\theta$ curve.

A similar argument applies to the eyeglasses graph. Again let $F_{j}$ be a region of maximal pressure so $k \geq 0$. By $(2), k \equiv 0$. If $F_{j}$ is the 4 -gon, this implies that all pressures are equal since the other two regions are adjacent to $F_{j}$. If $F_{j}$ is a monogon, then the 4-gon has the same maximal pressure and again all pressures are equal. Thus if $G_{0}$ does not degenerate to a figure eight, then it forms a geodesic net.

Finally we consider the possible pressures in the case that $G_{0}$ is a figure eight graph. Recall that each of the monogons has equal total curvature $K, 4 \pi / 3 \leq$ $K \leq 5 \pi / 3$, and the 2-gon region has total curvature $4 \pi-2 K$. The curvature $\bar{K}$ corresponds to a monogon target angle of $t_{i}=K-\pi, \pi / 3 \leq t_{i} \leq 2 \pi / 3$.

Claim 1. The pressure in the 2-gon region cannot be a strict minimum or maximum. 


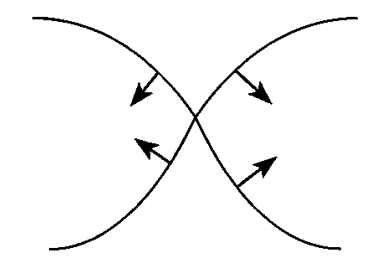

FiguRE 3. Curvatures when the 2-gon region has minimal pressure

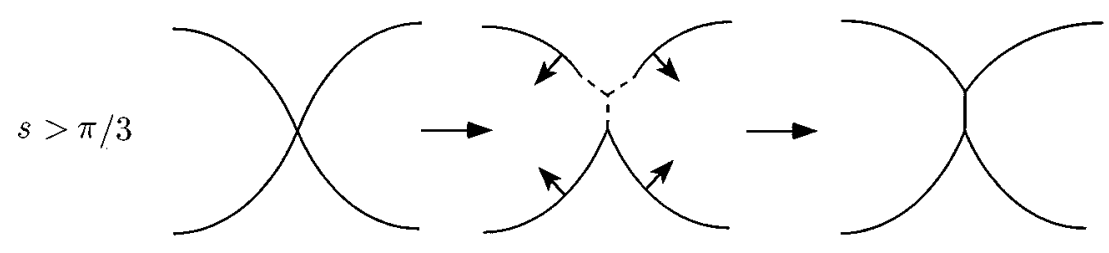

Figure 4. A length decreasing variation that preserves total curvatures

Proof of Claim 1. Assume that the 2-gon region has strictly smallest pressure. First consider the case that $K>4 \pi / 3$. Decrease the monogon target angle $t_{i}$ slightly, and thus decrease the targeted total curvature $K$ for the monogons. The new values are chosen to still be greater than $4 \pi / 3$, so that the new curve is still admissible. The arcs bounding the monogons near the vertex have non-zero curvature pointing into the monogons (see Figure 3 ), so $K$ can be reduced in both monogons by decreasing the length of $G_{0}$ near the vertex, a contradiction since we get a shorter admissible graph.

Suppose next that $K=4 \pi / 3$ and that $G_{0}$ has interior angle $s$ in the monogons, which have target value $\pi / 3$. Call the monogons $M_{1}$ and $M_{2}$ with pressure $\left(M_{1}\right) \geq$ pressure $\left(M_{2}\right)$. Since we are assuming that the 2-gon region has strictly smallest pressure we know that $k>0$ at points of $\partial M_{1}$ and $\partial M_{2}$ near the vertex. Since pressure $\left(M_{1}\right)$ is maximal, $k \geq 0$ on $\partial M_{1}$ and $k>0$ at any boundary point that separates $M_{1}$ from the 2 -gon. Applying (2) to $M_{1}$ gives that $s>t=\pi / 3$. We now perform a variation of $G_{0}$ in two steps, as in Figure 4. The first step replaces the degree-four vertex by two degree-three vertices, changing the graph into a $\theta$ graph and increasing the total curvature in each monogon region. Since $s>\pi / 3$ this step can be done so as to decrease the length of $G_{0}$ [M1, step 3 of 2.3]. In the second step, push the boundary of each monogon in slightly to restore its total curvature to the original value of $4 \pi / 3$, while decreasing the length of $G_{0}$ still further. This gives an admissible $\theta$ graph which is shorter than $G_{0}$, a contradiction. (If $s=\pi$ and the boundary curves coincide on an interval leaving the vertex, the second step is unnecessary.) If the 2-gon region has strictly maximal pressure, a similar argument yields a shorter admissible eyeglass graph and thus a contradiction.

Claim 1 implies that the monogon region $M_{1}$ has maximal pressure among the three regions, though perhaps not strictly maximal. We then have $k \geq 0$ on $\partial M_{1}$ and formula (2) implies that $s \geq t$, with equality only if $k \equiv 0$. Similarly Claim 1 implies that the monogon region $M_{2}$ has minimal pressure among the three regions, that $k \leq 0$ on $\partial M_{1}$ and that $s \leq t$ with equality only if $k \equiv 0$. This gives a contradiction unless $k \equiv 0$ and all the regions have the same pressure. In this 


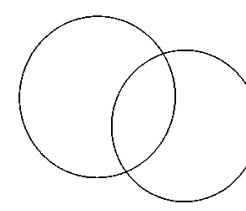

or

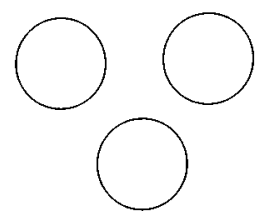

or

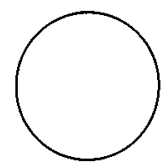

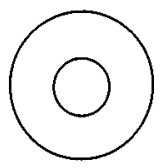

FiguRE 5. Three configurations of simple geodesics which divide the 2-sphere into four components

case all the boundary arcs are geodesics. Thus if the figure eight graph is lengthminimizing among admissible graphs, then it forms a geodesic net. This finishes the proof of Theorem 1 .

Corollary 1. There is a neighborhood of the round metric in the space of smooth metrics on the 2-sphere such that every metric in this neighborhood contains a geodesic net homeomorphic to a $\theta$ graph.

Proof. A geodesic monogon on the round metric is a great circle, which has an interior angle of $\pi$. Nearby metrics only have monogons with interior angle $>2 \pi / 3$, and therefore metrics near the round metric on $S^{2}$ do not contain a geodesic monogon of the type formed in a geodesic net of the type of an eyeglass graph. A metric near the round metric might contain a graph of the type of a figure eight, which degenerates to cover a geodesic on the round sphere. However a geodesic monogon on the round sphere has length $2 \pi$, so that geodesic nets of the type of a figure eight in nearby metrics have length close to $4 \pi$. The proof of Theorem 1 shows that the shortest admissible graph forms a geodesic net. On the round sphere a theta curve geodesic net has length $3 \pi$. In nearby metrics there is an admissible curve with length close to $3 \pi$. Thus the theta curve is the type of geodesic net produced by Theorem 1 .

The round sphere shows that one cannot hope to always find geodesic nets which are eyeglass graphs or figure eights.

Question 1. Do all metrics on the 2-sphere contain a geodesic net homeomorphic to a $\theta$ graph?

Question 2. Do the conclusions of Theorem 1 hold for arbitrary metrics on the 2-sphere?

We now consider the existence of geodesic nets dividing the 2-sphere into four regions. The approach of Theorem 1 breaks down: the number of combinatorial possibilities and the ways curves can bump up against each other get out of control. Nevertheless, we actually obtain a much stronger result in this case, without assuming positive curvature, though we do so by applying some deep results on the existence of closed geodesics on the 2-sphere.

Theorem 2. Let $S$ be a 2-sphere with an arbitrary smooth Riemannian metric. There exists a geodesic net $G$ partitioning $S$ into four components with one of the configurations shown in Figure 5.

Proof. A theorem originally stated by Lusternik and Schnirelman [L-S] states that in any metric on the 2-sphere there are at least three distinct simple closed geodesics. Various ways of proving this result now exist, including the method of flow by 


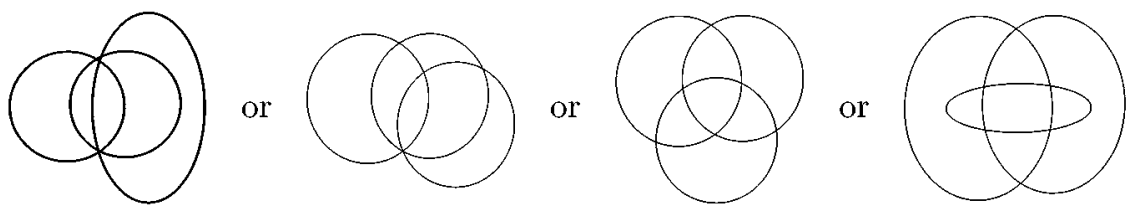

FiguRE 6. Four configurations of three curves in the 2-sphere

curvature. Grayson showed that if one takes the set of all round circles on the standard 2-sphere, considers the corresponding family of circles on $S$, and lets this family of circles evolve under the curvature flow, then there are at least three distinct geodesics which are the limit under the flow of different curves in the family $[G]$. Since in the initial family of closed curves no two intersect more than twice, and the curvature flow does not increase the number of intersections, any pair of the three resulting geodesics intersect in at most two points. If two of the three geodesics intersect, then they form a geodesic net of the first type. If all three geodesics are mutually disjoint, then they form a geodesic net of the second or third type.

Theorem 3. Let $S$ be a smooth, positively curved 2-sphere. Then there exists a geodesic net $G$ partitioning $S$ into four components having the first configuration of Figure 5. $S$ also admits a geodesic net partitioning $S$ into either 6,7 or 8 regions with one of the configurations in Figure 6.

Proof. The first configuration of Figure 5 is the only possibility for any pair of geodesics, since $S$ does not admit disjoint geodesics by the Gauss-Bonnet Theorem. The configurations of Figure 6 give the resulting embeddings of three simple closed curves in the 2-sphere, up to homeomorphism of the 2-sphere.

Conjecture 1. Let $S$ be a smooth Riemannian 2-sphere, $n$ a natural number. Then there is a geodesic net with vertices of degree 3 or 4 partitioning $S$ into $n$ regions.

This conjecture remains open even for the round 2-sphere.

Remark 1 . There are several natural ways to obtain geodesic nets on a closed surface $S$ of higher genus.

(a) A length-minimizing spline gives such a net. This spline is a shortest graph whose complement is a disk (or more generally any surface simpler than $S$ ). Existence of such objects is established in [Ch, Prop. in Sect. 4].

(b) Size-minimizing representatives of a one-dimensional homology class give geodesic nets with valence-three vertices (cf. [M2, Sect. 2]).

(c) Minimizers in a $Z_{3}$-homology class give geodesic nets with valence-three vertices.

\section{REFERENCES}

[A-T] F. J. Almgren, Jr., and Jean E. Taylor, The geometry of soap films and soap bubbles, Scientific American, July, 1976, 82-93.

[Ch] J. Choe, On the existence and regularity of fundamental domains with least boundary area, J. Diff. Geom. 29 (1989), 623-663. MR 91e:49040

[Cr] C. Croke, Poincaré's problem on the shortest closed geodesic on a convex hypersurface, J. Diff. Geom. 17 (1982), 595-634. MR 84f:58034 
[G] M. Grayson, Shortening embedded curves, Ann. of Math. 129 (1989), 71-112. MR 90a:53050

[H-M] J. Hass and F. Morgan, Geodesics and soap bubbles on surfaces (Math. Z., to appear).

$[\mathrm{He}]$ A. Heppes, Isogonal sphaerischen netze, Ann. Univ. Sci. Budapest Eötvös, Sect. Math. 7 (1964), 41-48. MR 30:3406

[Ho] H. Howards, Soap bubbles on surfaces, undergraduate thesis, Williams College, 1992.

[L-S] L. Lusternik and L. Schnirelman, Sur le probleme de trois géodesiques fermées sur les surface de genre 0, C. R. Acad. Sci. Paris 189 (1929), 269-271.

[M1] F. Morgan, Soap bubbles in $R^{2}$ and in surfaces, Pac. J. Math. 165 (1994), 347-361. MR 96a:58064

[M2] F. Morgan, Size-minimizing rectifiable currents, Invent. Math. 96 (1989), 333-348. MR 91b:49054

[T] J. E. Taylor, The structure of singularities in soap-bubble-like and soap-film-like minimal surfaces, Ann. of Math. 103 (1976), 489-539. MR 55:1208a

Department of Mathematics, University of California at Davis, Davis, California 95616

E-mail address: hass@math.ucdavis, edu

Department of Mathematics, Williams College, Williamstown, Massachusetts 01267

E-mail address: Frank.Morgan@williams.edu 